\title{
Pneumonia caused by extensive drug- resistant Acinetobacter baumannii among hospitalized patients: genetic relationships, risk factors and mortality
}

Yu jun $\mathrm{Li}^{1,2}$, Chu zhi $\mathrm{Pan}^{3+}$, Chang quan Fang ${ }^{4 \dagger}$, Zhu xiang Zhao ${ }^{2 \dagger}$, Hui ling Chen ${ }^{5 \dagger}$, Peng hao Guo ${ }^{6+}$ and Zi wen Zhao ${ }^{2^{*}}$

\begin{abstract}
Background: The clonal spread of multiple drug-resistant Acinetobacter baumannii is an emerging problem in China. We analysed the molecular epidemiology of Acinetobacter baumanni isolates at three teaching hospitals and investigated the risk factors, clinical features, and outcomes of hospital-acquired pneumonia caused by extensive drug-resistant Acinetobacter baumannii (XDRAB) infection in Guangzhou, China.

Methods: Fifty-two A. baumannii isolates were collected. Multilocus sequence typing (MLST) was used to assess the genetic relationships among the isolates. The bla OXA-51-like gene was amplified using polymerase chain reaction $(P C R)$ and sequencing. The resistance phenotypes were determined using the disc diffusion method. A retrospective case-control study was performed to determine factors associated with XDRAB pneumonia.

Results: Most of the $52 \mathrm{~A}$. baumannii isolates ( $N=37,71.2 \%)$ were collected from intensive care units (ICUs). The respiratory system was the most common bodily site from which $A$. baumannii was recovered $(N=45,86.5 \%)$. Disc diffusion classified the isolates into 17 multidrug-resistant (MDR) and 35 extensively drug-resistant (XDR) strains. MLST grouped the A. baumannii isolates into 5 existing sequence types (STs) and 7 new STs. ST195 and ST208 accounted for $69.2 \%$ (36/52) of the isolates. The clonal relationship analysis showed that ST195 and ST208 belonged to clonal complex (CC) 92. According to the sequence-based typing (SBT) of the bla OXA-51-like gene, 51 A. baumannii isolates carried OXA-66 and the rest carried OXA-199. There were no significant differences with respect to the resistance phenotype between the CC92 and non-CC92 strains $(P=0.767)$. The multivariate analysis showed that the APACHE II score, chronic obstructive pulmonary disease (COPD) and cardiac disease were independent risk factors for XDRAB pneumonia $(P<0.05)$. The mortality rate of XDRAB pneumonia was high (up to $42.8 \%$ ), but pneumonia caused by XDRAB was not associated with in-hospital mortality $(P=0.582)$.

Conclusions: ST195 may be the most common ST in Guangzhou, China, and may serve as a severe epidemic marker. SBT of bla OXA-51-like gene variants may not result in sufficient dissimilarities to type isolates in a small-scale, geographically restricted study of a single region. XDRAB pneumonia was strongly related to systemic illnesses and the APACHE II score but was not associated with in-hospital mortality.
\end{abstract}

Keywords: Acinetobacter baumannii, Extensive drug resistance, Multilocus sequence typing, bla OXA-57-like gene, Pneumonia

\footnotetext{
* Correspondence: zhaozw2016@163.com

${ }^{\dagger}$ Equal contributors

2Department of Respiratory Medicine, Guangzhou First People's Hospital,

Guangzhou Medical University, Panfu Road, Guangzhou, China

Full list of author information is available at the end of the article
} 


\section{Background}

Acinetobacter baumannii (AB) is one of the most important and common pathogens causing nosocomial outbreaks worldwide, especially in intensive care units (ICUs). The most common bodily site of $A$. baumannii infection is the respiratory tract, particularly in cases of hospital-acquired pneumonia (HAP) [1, 2]. A. baumannii is also notorious for its remarkable ability to acquire antibiotic resistance. Data from the CHINET surveillance system demonstrated that $A$. baumannii resistance to many important antimicrobial agents has increased, especially imipenem and meropenem, which increased from $31 \%$ in 2005 to $62.4 \%$ in 2014 and from $39 \%$ in 2005 to $66.7 \%$ in 2014, respectively [3]. Recently, the rise in the frequency of nosocomial infections caused by extremely drug resistant (XDR) A. baumannii strains (defined as resistance to all available antibiotics except colistin and tigecycline) has been of great concern because XDR resistance has been associated with high mortality and treatment failure [2, 4-7]. According to our previous study [5], most of the isolates $(76.2 \%, 32$ / 42) were XDR strains, mostly recovered from the respiratory system, but at present little research concerning extensive drug-resistant $A$. baumannii (XDRAB) pneumonia has been reported.

Currently, A. baumannii is recognized as one of the most difficult health care-associated infections to control and treat, and the optimal treatment of infections caused by XDRAB has not been established [6]. Surveillance of A baumannii isolates may inform prevention and control measures for these infections. Additionally, determining the process of disease spread by routine surveillance can abrogate routes of bacterial transmission [2]. Multilocus sequence typing (MLST) is a widely used technique for bacterial typing. MLST provides a portable method that is suitable for global epidemiological studies and monitoring of the national and international spread of bacteria [8, 9]. Currently, two large national studies $[10,11]$ have confirmed that CC92 represents the most epidemic sequence type (ST) in China. ST92, which is the founder of CC92, is the predominant ST, whereas other STs belonging to CC92 vary by area. ST75 may be the most common epidemic ST in eastern China [12], whereas ST138 may be the most common ST in western China [13]. Our previous study discovered that ST195 and ST208 belonged to CC92 were the major clone spreading in our hospital [5]. We assumed that ST195 and ST208 may be more common in southern China Guangzhou area, but this needed to be confirmed further. These differences may be due to the different antibiotic usage habits, which possibly influenced the evolution of ST92. However, little is known about the relationship between antibiotic resistance and certain STs. Although MLST has many advantages, it is a robust scheme that is often time-consuming, expensive, and labour-intensive [14]. Currently, several studies have reported that sequence-based typing (SBT) of bla $a_{O X A-51-l i k e}$ gene variants has potential for application to assess the epidemiological characterization of A. baumannii [14-16], but more data are needed.

This study investigated $52 \mathrm{~A}$. baumannii isolates from three teaching hospitals in Guangzhou to determine the clonality of the isolates. A case-control study was conducted to evaluate the characteristics, risk factors and outcomes for hospital-acquired XDRAB pneumonia, and the relationship between antibiotic resistance and certain STs was also investigated.

\section{Methods}

Bacterial isolates and antimicrobial susceptibility testing From April 2011 to February 2012, A total of 52 A. baumannii isolates were collected as part of the standard patient care regimen from three teaching hospitals (Guangzhou First People's Hospital, Guangzhou Medical University, Panfu Road, Guangzhou, China; the Third Affiliated Hospital of Sun Yat-sen University, Tian He Road, Guangzhou, China; and the First Affiliated Hospital of Sun Yat-sen University, Zhong Shan Er Road, Guangzhou, China). Among the $52 \mathrm{~A}$. baumannii isolates, 42 isolates had been reported in our previous study [5]. All A. baumannii isolates derived from clinical samples (sputum,bronchoalveolar lavage fluid, blood, cerebrospinal fluid, and urine) were collected from patients hospitalized in the general wards and intensive care units (ICUs), Duplicate isolates from the same patients were excluded. The Vitek 2 (bioMerieux, Inc., Durham, NC, USA) automated microbiology system was used in identification of isolates.

According to Clinical and Laboratory Standards Institute (CLSI; M100-S22, 2012) [17], disc diffusion method were used to detect the susceptibility of $52 \mathrm{~A}$. baumannii isolates against 15 antibiotics to determine the resistance phenotype. Isolates that showed resistance or intermediate susceptibility to imipenem, meropenem, amikacin, piperacillin/tazobactam, cefoperazone/sulbactam, ceftazidime, ceftriaxone, cefepime, aztreonam, levofloxacin, ciprofloxacin, doxycycline and tobramycin were considered XDRAB isolates. Melone Pharmaceutical Co. Ltd. (China) provided the antibiotic discs (OXOID). Escherichia coli ATCC 25922 and Pseudomonas aeruginosa ATCC 27853 were used as the control organisms.

\section{Molecular epidemiological typing}

Multilocus sequence typing (MLST) was performed on A. baumannii, according to Bartual et al. [18]. Seven conserved housekeeping genes (gltA, gyrB, gdhB, recA, cpn60, gpi, and rpoD) were amplified and sequencing. The allelic numbers and sequence types (STs) were 
identified by means of the Pubmlst database [19]. The eBURST algorithm (version 3) [20] was used to assign STs to clonal complexes (CCs) and to assess the genetic relationships among the sequences. Sequence-based typing of the bla OXA-51-like genes (SBT- bla OXA-51-like genes) was carried out as follows. The OXA-69A and OXA-69B primers [21], which were external to the bla ${ }_{O X A-51-l i k e}$ gene, were used to amplify the entire gene sequence, followed by sequencing. The sequences were analysed using BLAST (https://blast.ncbi.nlm.nih.gov/ Blast.cgi) to determine the genetic diversity of the bla $a_{O X-51-l i k e}$ genes $[14,15]$.

\section{Case-control study}

A retrospective case-control study was performed to evaluate the characteristics, risk factors and outcomes of hospital-acquired XDRAB pneumonia. The cases included patients from whom a XDRAB isolate was isolated from clinical cultures of respiratory secretions and who had been shown to have hospital-acquired pneumonia, including ventilator-associated pneumonia (VAP) defined as pneumonia that occurred more than $48 \mathrm{~h}$ after endotracheal intubation [22].

The inclusion criteria consisted of the following: a) diagnosis of pneumonia [23] the presence of new or progressive pulmonary infiltrates in chest radiographs, plus at least two of the following supportive clinical signs: temperature of $>38{ }^{\circ} \mathrm{C}$ or $<35.5^{\circ} \mathrm{C}$, leukocytosis $(>12,000$ $\left.\mathrm{WBC} / \mathrm{mm}^{3}\right)$ or leukopenia $\left(<4000 \mathrm{WBC} / \mathrm{mm}^{3}\right)$, purulent bronchial secretions, or worsening oxygenation and $\mathrm{b}$ ) at least two positive respiratory samples for A. baumannii : protected specimen brushing cultures $10^{3} \mathrm{cfu} / \mathrm{mL}$, bronchoalveolar lavage (BAL) fluid specimen $10^{4}$ or $10^{5} \mathrm{cfu} /$ $\mathrm{mL}$, or $10^{6} \mathrm{cfu} / \mathrm{mL}$ in an endotracheal aspirate [23]. Patients $<18$ years of age, patients hospitalization for $<48 \mathrm{~h}$ and patients with incomplete medical records were excluded from this study, as were patients who had other non- $A$. baumannii -positive cultures in addition to $A$. baumannii to avoid the inclusion of $A$. baumannii colonization.

The controls were randomly selected adult inpatients in the participating hospitals who were diagnosed with nonXDRAB hospital-acquired pneumonia during their hospital stay. The controls were matched to the cases by hospital. Two controls were recruited for each case. Computerized medical, pharmaceutical and microbiological records were reviewed. A specially designed case record form was used to collect demographic and clinical data, including age, gender, underlying diseases, severity of diseases [calculated by the Acute Physiology and Chronic Health Evaluation (APACHE) II score] while admitted to the general wards or ICU, invasive procedures (central venous and/or arterial catheter, urinary catheter, nasogastric tube and mechanical ventilation), duration of stay in the ICU, hospital stay and antibiotic exposure.

\section{Statistical analysis}

Categorical variables were compared using the Chi-square test with Yates correction or Fisher's exact test. Continuous variables were analysed using the test. A $P$ value $<0.05$ in a two-tailed test was considered statistically significant. To test the independence of the risk factors for XDRAB pneumonia, significant variables $(P<0.05)$ in the univariate analyses were entered into a multivariate logistic regression model. SPSS (version 18.0) was used for all calculations.

\section{Results}

Characteristics of the $52 \mathrm{~A}$. baumannii isolates

Most of the isolates $(N=37,71.2 \%)$ were obtained from intensive care units (ICUs). The respiratory system was the most common bodily site from which $A$. baumannii was recovered $(N=45,86.5 \%)$, followed by the blood ( $N$ $=3,5.8 \%$ ). Disc diffusion testing (supplementary information) classified the $52 \mathrm{~A}$. baumannii isolates into 17 MDR and 35 XDR strains (Table 1).

\section{MLST and SBT- bla $a_{O X-51-l i k e}$ genes}

According to the MLST, $52 \mathrm{~A}$. baumannii isolates were grouped into 12 distinct STs, including 5 existing STs and 7 novel STs (STn1 to STn7). STn4 carried allele G1 with a $\mathrm{T} \rightarrow \mathrm{C}$ mutation at the 3rd nucleotide site (nt3) on the gpi111 locus. STn5 carried allele A1 with an $\mathrm{A} \rightarrow \mathrm{C}$ mutation at nt156 and nt159 on the gltA1 locus. ST195 and ST208 were the most common STs, accounting for $69.2 \%$ of all isolates. The clonal relationship analysis showed that ST195 and ST208 belonged to CC92. According to the sequence-based typing of the $b l a_{O X A-}$ 51-like genes, 51 A. baumannii isolates carried OXA-66 and the rest carried OXA-199. No significant differences with respect to the resistance phenotype were detected between the CC92 and non-CC92 strains $(P=0.767)$ (Tables 1 and 2 and Fig. 1).

\section{Case-control study}

Full medical records were available for 42 of the 52 patients from whom the A. baumannii isolates were isolated. A total of 32 patients were diagnosed with $\mathrm{XDRAB}$ acquisition, and 21 patients finally met the inclusion criteria and were assessed in the case-control study. Of the 21 patients with XDRAB pneumonia, 17 were men and four were women. The mean age was 77.5 years (standard deviation 11.6 years). Sixteen patients were receiving care in ICUs and 5 were in general wards during specimen collection (Tables 1 and 3).

The potential risk factors for patients with XDRAB pneumonia are shown in Table 3. The two groups were 


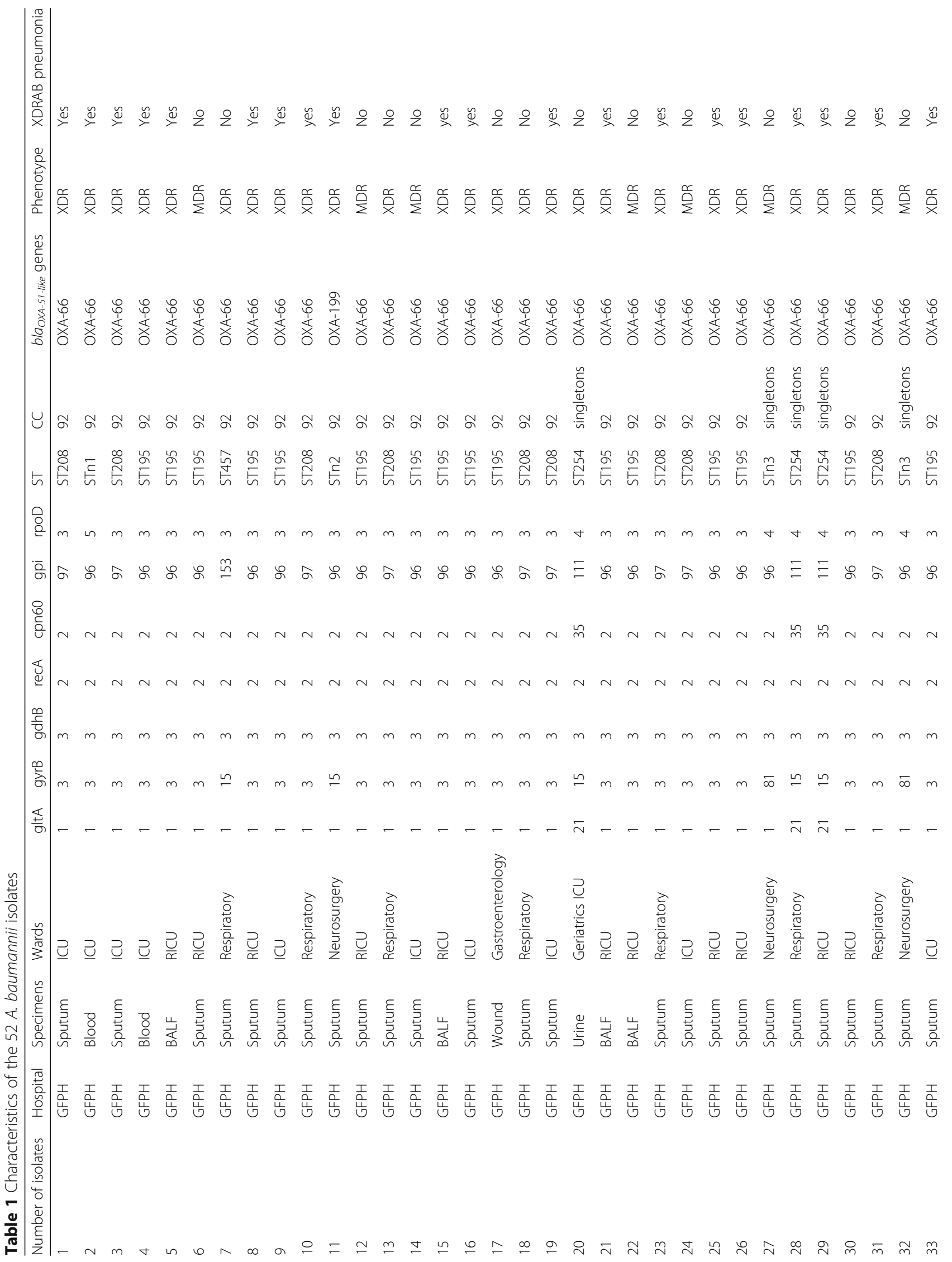




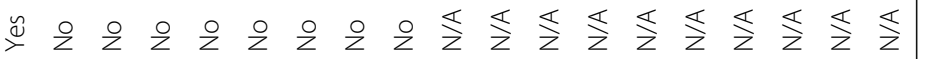

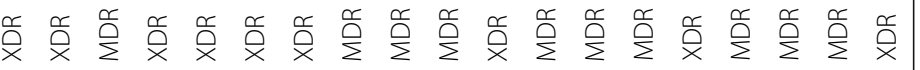

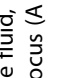

o

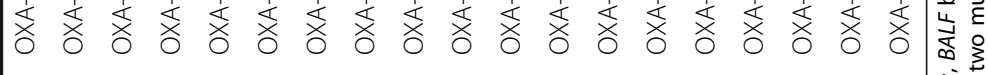

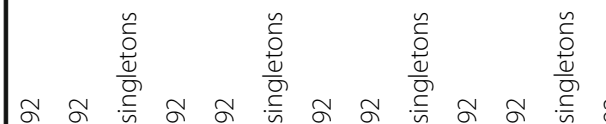

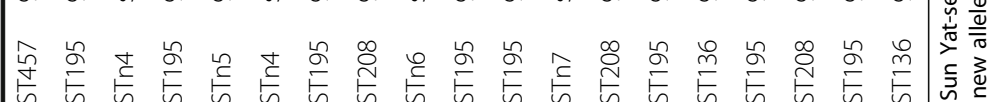

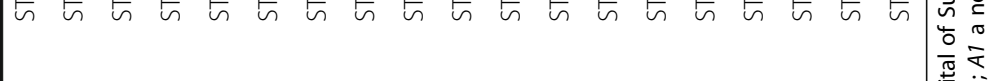

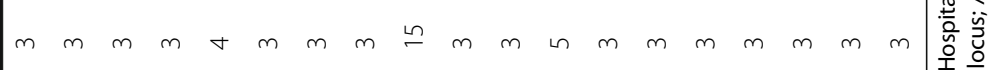

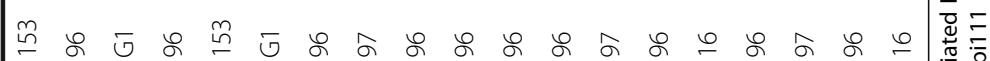

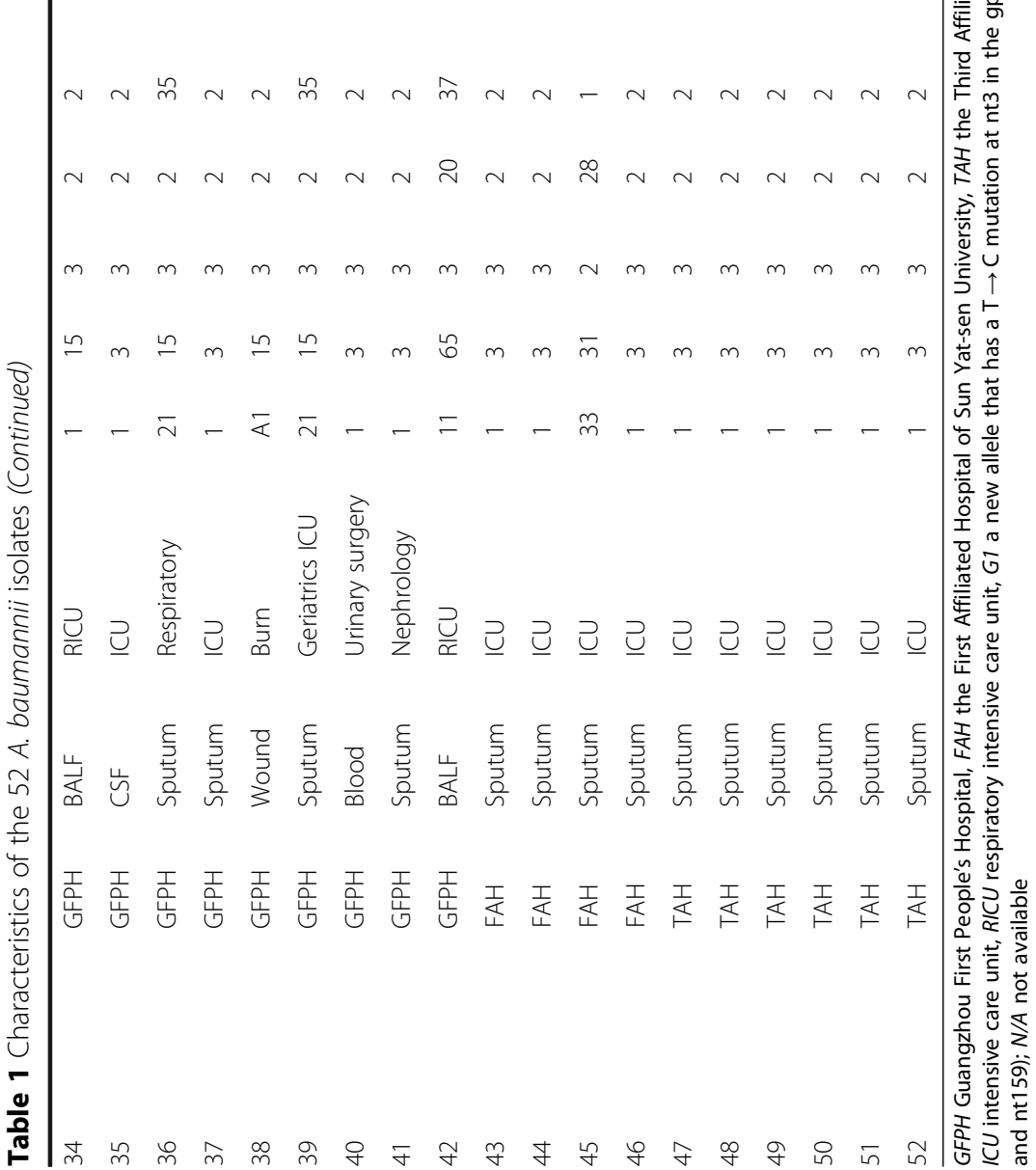


Table 2 The relationship between the clonal complex (CC) and resistance phenotype of the $52 \mathrm{~A}$. baumannii isolates

\begin{tabular}{|c|c|c|c|c|c|}
\hline \multirow[t]{2}{*}{ Clonal complex } & \multirow{2}{*}{$\begin{array}{l}\text { No. } \\
\text { isolates }\end{array}$} & \multirow{2}{*}{$\begin{array}{l}\text { MDR } \\
\text { N (\%) }\end{array}$} & \multirow{2}{*}{$\begin{array}{l}\text { XDR } \\
\text { N (\%) }\end{array}$} & \multicolumn{2}{|c|}{ Statistical analysis ${ }^{a}$} \\
\hline & & & & $x^{2}$-values & $P$ values \\
\hline $\mathrm{CC92}$ & 43 & $14(32.6)$ & $29(67.4)$ & 0.088 & 0.767 \\
\hline ST195 & 24 & 9 & 15 & & \\
\hline ST208 & 12 & 5 & 7 & & \\
\hline ST457 & 2 & 0 & 2 & & \\
\hline ST136 & 2 & 0 & 2 & & \\
\hline $\operatorname{STn} 1$ & 1 & 0 & 1 & & \\
\hline $\operatorname{STn} 2$ & 1 & 0 & 1 & & \\
\hline $\operatorname{STn} 5$ & 1 & 0 & 1 & & \\
\hline Non-CC92 & 9 & $4(44.4)$ & $5(55.6)$ & & \\
\hline ST254 & 3 & 0 & 3 & & \\
\hline $\operatorname{STn} 3$ & 2 & 2 & 0 & & \\
\hline STn4 & 2 & 0 & 2 & & \\
\hline STn6 & 1 & 1 & 0 & & \\
\hline STn7 & 1 & 1 & 0 & & \\
\hline
\end{tabular}

${ }^{a}$ comparison of $\mathrm{CC} 92$ with non-CC92 strains

similar with respect to gender, days of mechanical ventilation before XDRAB pneumonia, days spent in the hospital before XDRAB pneumonia, length of stay in the ICU, and length of stay in the hospital. Additionally, no significant differences were detected with respect to malignancy, renal disease, neurological disease, urinary catheter, nasogastric tube, mechanical ventilation, or the use of glucocorticoids, PPIs, cephalosporin, $\beta$-lactamase inhibitor, quinolone or minoglycoside. The mortality rate of XDRAB pneumonia was high (up to $42.8 \%$ ), but pneumonia caused by XDRAB was not associated with in-hospital mortality $(P=0.582)$. Compared with the non-XDRAB patients, the patients with XDRAB pneumonia were significantly more likely to be older $(77.5 \pm$ 11.6 vs $68.6 \pm 18.4$ years, $P=0.023$ ) and have a higher initial severity of illness at admission as indicated by the higher APACHE II score $(21.9 \pm 6.8$ vs $18.0 \pm 4.9, P=$ $0.011)$. Moreover, chronic obstructive pulmonary disease (COPD), cardiac disease and carbapenem use were risk factors for XDRAB pneumonia $(P<0.05)$.

The multivariate analysis using a logistic regression model results are presented in Table 4. The APACHE II score (OR, 1.17; 95\% CI: 1.01-1.35, $P=0.034)$, COPD (OR, 7.25; 95\% CI: $1.54-33.9, P=0.012$ ), and cardiac disease (OR, 6.94; 95\% CI: 1.43-33.6, $P=0.016$ ) were identified as independent risk factors for XDRAB acquisition.

\section{Discussion}

In this study, we examined the molecular typing and characteristics of A. baumannii at three teaching hospitals. In the MLST, CC92 was the most prevalent clonal complex. However, ST92, which was the predicted founder of CC92 and was reported to be one of the most epidemic STs in multiple provinces in China $[1,10,11]$, was not detected in our study. ST92 was also not

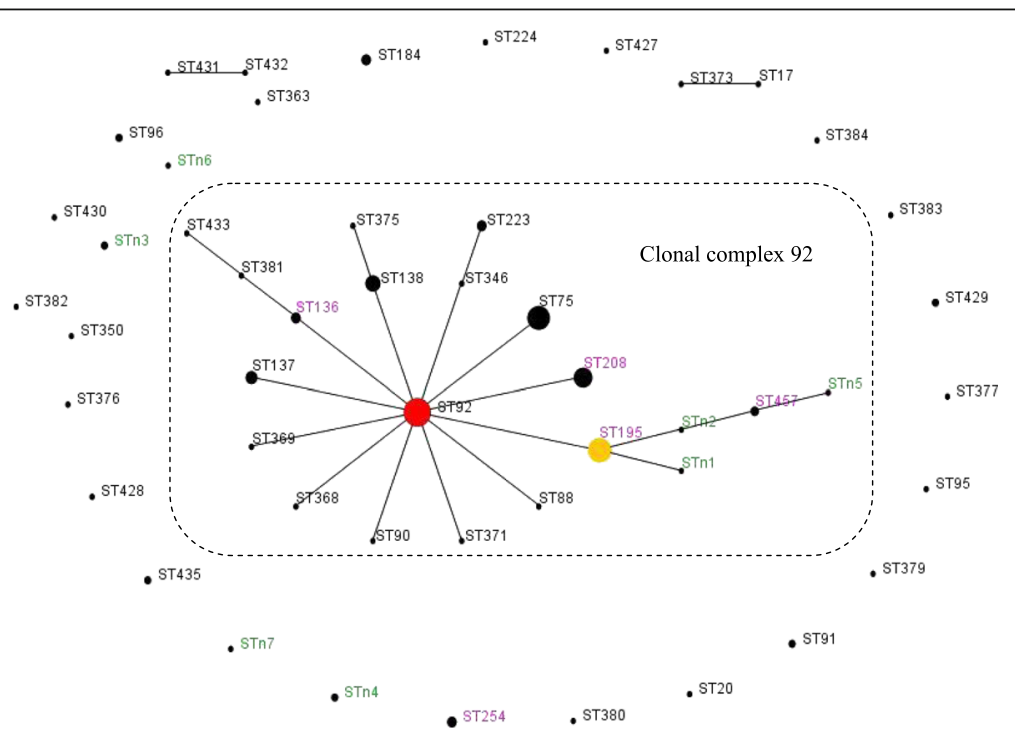

Fig. 1 Population snapshot of A. baumannii in this study and other existing isolates in China. Population snapshot of A. baumannii in this study and existing isolates in China based on the data contained in the Pubmlst database as of 27 April $2013[5,18]$ represented by an eBURST algorithm. Circles represent STs, and their sizes correspond to the numbers of isolates. The red circle represents the founder ST (ST92). The broken line indicates clonal complex (CC) 92. The ST labels are coloured as follows: black, STs found only in the Pubmlst database; green, STs found only in this study; and purple, STs found in both the Pubmlst database and this study. ST254, STn3, STn4, STn6 and STn7 were the singletons in this study 
Table 3 Comparison of clinical data for pneumonia-related characteristics in HAP patients with XDRAB and non-XDRAB

\begin{tabular}{|c|c|c|c|}
\hline & $\begin{array}{l}\text { XDRAB } \\
(N=21) \\
N(\%)\end{array}$ & $\begin{array}{l}\text { Non-XDRAB } \\
(N=42) \\
N(\%) \\
\end{array}$ & $P$-value \\
\hline Age, $y^{a}$ & $77.5 \pm 11.6$ & $68.6 \pm 18.4$ & 0.023 \\
\hline Gender (M/F), n & $17 / 4$ & 23/19 & 0.079 \\
\hline APACHE II score ${ }^{a}$ & $21.9 \pm 6.8$ & $18.0 \pm 4.9$ & 0.011 \\
\hline Related to hospitalization ${ }^{a}$ & $18(85.7)$ & $30(71.4)$ & 0.347 \\
\hline Days of mechanical ventilation before XDRAB (days) & $10.5 \pm 11.6$ & $5.2 \pm 5.8$ & 0.059 \\
\hline Hospital days before XDRAB (days) & $18.3 \pm 11.3$ & $12.6 \pm 11.2$ & 0.064 \\
\hline Length of stay in the ICU (days) & $30.1 \pm 20.0$ & $21.4 \pm 21.7$ & 0.127 \\
\hline Length of stay in the hospital (days) & $45.5 \pm 28.8$ & $38.5 \pm 24.2$ & 0.199 \\
\hline \multicolumn{4}{|l|}{ Associated disease, n (\%) } \\
\hline COPD & $13(61.9)$ & $9(21.4)$ & 0.001 \\
\hline Diabetes mellitus & $2(9.5)$ & $10(40.4)$ & 0.307 \\
\hline Malignancy & $2(9.5)$ & $9(21.4)$ & 0.411 \\
\hline Cardiac disease & $13(61.9)$ & $6(14.2)$ & 0.000 \\
\hline Renal disease & $5(23.8)$ & $2(4.7)$ & 0.065 \\
\hline Neurological disease & $8(38.0)$ & $22(52.3)$ & 0.422 \\
\hline \multicolumn{4}{|l|}{ Device, n (\%) } \\
\hline Urinary catheter & $21(100.0)$ & $36(85.7)$ & 0.172 \\
\hline Nasogastric tube & $21(100.0)$ & $36(85.7)$ & 0.172 \\
\hline Mechanical ventilation & $16(76.1)$ & $31(73.8)$ & 1.000 \\
\hline \multicolumn{4}{|l|}{ Drug usage, n (\%) } \\
\hline Glucocorticoids & $10(47.6)$ & $18(42.8)$ & 0.720 \\
\hline PPIs & $14(66.7)$ & $32(76.1)$ & 0.422 \\
\hline \multicolumn{4}{|l|}{ Antimicrobial n (\%) } \\
\hline \multicolumn{4}{|l|}{ Cephalosporin } \\
\hline Second generation & $3(14.2)$ & $5(11.9)$ & 1.000 \\
\hline Third generation & $7(33.3)$ & $18(42.8)$ & 0.649 \\
\hline$\beta$-lactamase inhibitor & $20(95.2)$ & $31(73.8)$ & 0.089 \\
\hline Quinolone & $13(61.9)$ & $19(45.2)$ & 0.327 \\
\hline Aminoglycoside & $2(9.5)$ & $4(9.5)$ & 1.000 \\
\hline Carbapenem & $11(52.3)$ & $11(26.1)$ & 0.040 \\
\hline Antimicrobial Combination therapy, n (\%) & $11(52.3)$ & $19(45.2)$ & 0.593 \\
\hline Mortality, n (\%) & $9(42.8)$ & $15(35.7)$ & 0.582 \\
\hline
\end{tabular}

${ }^{a}$ Values are presented as the mean \pm standard deviation; malignancy includes haematological malignancies and solid tumours; cardiac disease includes coronary artery disease, hypertensive heart disease, valvular disease and cardiomyopathy; renal disease includes chronic renal failure; neurological disease includes cerebral haemorrhage and cerebral infarction; PPIs proton pump inhibitor drugs

Table 4 Multi-variate analysis of risk factors for patients with XDRAB pneumonia

\begin{tabular}{llc}
\hline Risk factor & OR $(95 \% \mathrm{Cl})$ & $P$-value \\
\hline APACHE II score & $1.17(1.01-1.35)$ & 0.034 \\
COPD & $7.25(1.54-33.9)$ & 0.012 \\
Cardiac disease & $6.94(1.43-33.6)$ & 0.016 \\
\hline
\end{tabular}

detected in other studies from China [1, 5] and South Africa [24]. The lack of ST92 detection may be due to the relatively small sample size in our study, which may not accurately represent the diversity and relative abundance of $A$. baumannii STs. However, some studies $[1,5,25]$ have shown that ST195, ST208, ST365, and ST191 (but not ST92) are the most common STs discovered in different hospitals across China. In this study, ST195 was the most commonly observed ST, accounting for $24 / 52$ (46.1\%) of the isolates, follow by ST208. These 
findings are similar to the findings of Zhou et al. [25], who found that ST195 accounted for 31/57 (54.4\%) isolates. These data collectively suggest that ST195 is the most common ST and may serve as a severe epidemic marker in the Guangzhou area.

Some data are available to clarify why CC92 is predominant. Zhong et al. [12] found that ST75, which is the single locus variant (SLV) of ST92, differs in its gpi loci and belongs to CC92, has more severe imipenem resistance that might improve its chances of survival. In our study, CC92 accounted for $82.7 \%(43 / 52)$ of the isolates. Most of the XDR isolates $(29 / 34,85.3 \%)$ were from CC92. However, CC92 included 14 MDR isolates with less severe resistance, and non-CC92 had similar results. No significant differences with respect to the resistance phenotype were detected between the CC92 and nonCC92 strains $(P=0.767)$. Runnegar et al. [26] revealed that CC92 had been spread in the hospital for 9 years with variable antibiotic susceptibility. These findings may suggest that both adaptation to the hospital environment and antibiotic resistance have been important for the success of CC92.

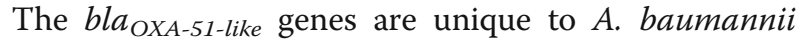
and immobile and thus may be used as markers for the identification of this species [21]. Recently, sequencebased typing (SBT) of bla $a_{O X A-51-l i k e}$ gene variants was reported to have potential for use in the epidemiological characterization of $A$. baumannii isolates obtained from various locations in Europe. $b l a_{O X A-69}, b l a_{O X A-66}$, and $b l a_{O X A-71}$ are the predominant members of closely related bla $a_{O X A-51-l i k e}$ subgroups, which are associated with European clone I (EUI), EUII, and EUIII, respectively [14-16]. However, few studies have investigated the correlation between bla OXA-51-like variants with MLST typing.

According to a study by Hamouda et al. [14], MLST data showed that all isolates harbouring the major

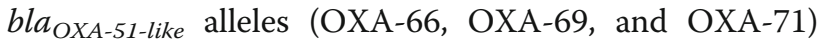
fell within the three major European clonal lineages. The SBT-bla $a_{\text {OXA-51-like }}$ gene scheme produced results comparable to those produced by the Bartual MLST for the identification of the major epidemic lineages. Pournaras et al. [27] evaluated the SBT-bla $a_{\text {OXA-51-like }}$ gene scheme in parallel with Pasteur's MLST. In the study, according to the SBT-bla OXA-51-like gene, all 585 A. baumannii isolates from a large international collection were typed and assigned correctly to the nine CCs and the singleton ST78. Zhou et al. [25] revealed that 52 isolates from Guangzhou city in China carrying bla $O X A-66$ were assigned to six distinct STs, which clustered into CC92; the remaining isolates belonged to four singletons that each carried a single bla OXA-51-like allele. The results of these studies indicate that the SBT-bla $a_{O X A-51-l i k e}$ gene scheme has the advantage of a significantly reduced sequencing cost and assay time and may be effective for the rapid typing of $A$. baumannii strains. However, in our study, only one isolate carried OXA-199, whereas the remaining $51 \mathrm{~A}$. baumannii isolates carried OXA- 66 . The SBT-bla ${ }_{O X A-51-l i k e}$ gene scheme failed to discriminate strains carrying the same OXA-66 allele. Similar findings reported by Wang et al. [28] showed that 18 representative isolates from different hospitals across China carried the same OXA-66 allele.

In Hamouda et al.'s study [14], the SBT-bla OXA-51-like method was evaluated in a large international collection of A. baumannii isolates including 22 countries. Similarly, Pournaras et al. [20] typed isolates obtained from various locations in Europe (Italy, Greece, Turkey, and Lebanon). Environmental differences among countries may act as natural selection forces [12] to introduce diversity among bla ${ }_{O X A-51-l i k e}$ genes. When confined to one city or hospital, the controlled environment and antibiotic usage habits might influence the convergent evolution of the bla $a_{O X A-51-l i k e}$ genes. As a result, singlelocus sequence-based typing of the bla OXA-51-like genes may not be effective at distinguishing isolates in a smallscale, geographically restricted study. However, further investigation is needed to confirm this hypothesis.

Some studies have reported risk factors for antibiotic resistance in A. baumannii infection, which include the length of stay in an ICU, severity of the underlying disease, mechanical ventilation, invasive procedures, and prior antibiotic use [29-32]. However, the clinical characteristics of XDRAB pneumonia have rarely been reported. In our study, univariate analysis was used to identify patients at risk of acquiring XDRAB pneumonia and showed that patients with XDRAB were older and had higher APACHE II scores than patients without XDRAB, which was similar to the findings of Özgür et al. [33]. Additionally, patients who had COPD and cardiac diseases were more likely to acquire XDRAB. Multivariate analysis using a logistic regression model showed that the APACHE II score, COPD and cardiac disease were independent risk factors for XDRAB pneumonia development. Thus, XDRAB may be particularly pathogenic in patients who are immunocompromized. Our study found that carbapenem use was an important risk factor for XDRAB pneumonia $(P=0.04)$. Previous studies $[29,31,34]$ have also shown that selective pressure exerted by the use of carbapenem leads to the emergence of multidrug resistant (MDR) and XDR A. baumannii isolates. Therefore, rational use of carbapenem is necessary to reduce the risk of generating resistant mutants.

High mortality rates have been reported for nosocomial pneumonia caused by $A$. baumannii (ranging from 28.1 to $85.3 \%$ ). The independent risk factors included the severity of illness (e.g., severe sepsis, septic shock 
and APACHE II score) and empiric inappropriate therapy $[31,33,35,36]$. In one study, patients with carbapenem-resistant $A$. baumannii (CRAB) pneumonia had a higher mortality rate than patients with carbapenem-susceptible $A$. baumannii (CSAB) pneumonia based on the survival analysis $(29.9 \%$ vs $45.6 \%$, respectively, $P=0.02$ ) [31]. However, another study reported mortality rates for patients with MDR A. baumannii infections that were not significantly higher than the mortality rates in patients without MDR A. baumannii infections [37]. Furthermore, in a study on ventilator-associated pneumonia (VAP) due to XDRAB, the mortality rates were not significantly higher than those of non-XDRAB VAP in ICU patients [33]. Our study showed a high hospital mortality rate in patients with XDRAB pneumonia, but $A$. baumannii resistance was not associated with mortality $(P=0.582)$.

\section{Conclusions}

ST195 may be the most common ST in the Guangzhou region of China and may serve as a severe epidemic marker in this region. SBT of bla $a_{O X A-51-l i k e}$ gene variants may not be able to sufficiently distinguish isolates obtained from a small-scale, geographically restricted study. XDRAB pneumonia was strongly related to systemic illnesses and the APACHE II score but was not associated with in-hospital mortality.

\section{Additional file}

Additional file 1: 52 Isolates were tested against antibiotics by disc diffusion. All 52 of the isolates were tested against a panel of antibiotics with the disc diffusion method, as recommended by the Clinical and Laboratory Standards Institute (CLSI;M100-S22, 2012) [17], to determine the resistance phenotype. Multidrug resistance (MDR) was defined as acquired non-susceptibility to at least one agent in three or more antimicrobial categories, and extensive drug resistance (XDR) was defined as resistance to all available antibiotics except colistin and tigecycline [5]. GFPH, Guangzhou First People's Hospital; FAH, the First Affiliated Hospital of Sun Yat-sen University; TAH, the Third Affiliated Hospital of Sun Yat-sen University; R, resistance; S, sensitivity; I, intermediate; PB, polymyxin B (300 units); TGC, tigecycline $(15 \mu \mathrm{g})$; IPM, imipenem $(10 \mu \mathrm{g}) ; A K$, amikacin $(30 \mu \mathrm{g}) ; \mathrm{TOB}$, tobramycin $(10 \mu \mathrm{g}) ;$ MEM, meropenem $(10 \mu \mathrm{g}) ;$ TZP, piperacillin/tazobactam (100 / $10 \mu \mathrm{g})$; SCF, cefoperazone/sulbactam (70 / $35 \mu \mathrm{g})$; CAZ, ceftazidime $(30 \mu \mathrm{g}) ;$ CRO, ceftriaxone $(30 \mu \mathrm{g}) ; \mathrm{FEP}$, cefepime $(30 \mu \mathrm{g}) ;$ ATM, aztreonam $(30 \mu \mathrm{g}) ; \mathrm{LEV}$, levofloxacin $(5 \mu \mathrm{g}) ; \mathrm{CIP}$, ciprofloxacin $(5 \mu \mathrm{g}) ; \mathrm{DO}$, doxycycline $(30 \mu \mathrm{g})$. (DOCX $24 \mathrm{~kb})$

\footnotetext{
Abbreviations

AB: Acinetobacter baumannii; APACHE II score: Acute Physiology and Chronic Health Evaluation II score; ATS/IDSA: American Thoracic Society/Infectious Diseases Society of America; BAL: Bronchoalveolar lavage; CC: Clonal complex; CLSI: Clinical and Laboratory Standards Institute; COPD: Chronic obstructive pulmonary disease; CRAB: Carbapenem-resistant A. baumannii; CSAB: Carbapenem-susceptible A. baumannii; ICUs: Intensive care units; MDR: Multidrug resistant; MLST: Multilocus sequence typing; PCR: Polymerase chain reaction; SBT: Sequence-based typing; ST: Sequence type; VAP: Ventilator-associated pneumonia; XDRAB: Extensive drug-resistant Acinetobacter baumannii
}

\section{Acknowledgements}

We thank Hui fen YE, of the Department of Clinic Laboratory, Guangzhou First People's Hospital, for technical support in culturing and reisolating strains.

\section{Funding}

This work was funded by the Guangzhou Medical and Health Science and Technology Project (no. 20151A011004).

\section{Availability of data and materials}

All data generated or analysed during this study are included in this published article and its supplementary information files. Additional file 1: 52 Isolates were tested against antibiotics by disc diffusion. The file's format is word document, the file contains susceptibility data of $52 \mathrm{~A}$. baumannii isolates against 15 antibiotics, which were used to determine the resistance phenotype.

\section{Authors' contributions}

YjL supervised the study, performed the susceptibility testing and MLST, and wrote the manuscript. CZP discussed the data and helped finalize the manuscript. $\mathrm{CqF}$ contributed to the case-control study and the statistical analysis. ZxZ contributed to the susceptibility testing and sequence-based typing of the bla $a_{0 X A-}$ 51-like genes. HIC and PhG provided advice regarding the susceptibility testing technology. ZwZ planned and supervised the experiments. All authors read and approved the final manuscript.

\section{Competing interests}

The authors declare that they have no competing interests.

\section{Consent for publication}

Not applicable.

\section{Ethics approval and consent to participate}

The Guangzhou First People's Hospital Ethics Committee approved the study, and all of the patients provided informed consent for inclusion in the study.

\section{Publisher's Note}

Springer Nature remains neutral with regard to jurisdictional claims in published maps and institutional affiliations.

\section{Author details}

${ }^{1}$ The First Affiliated Hospital of Jinan University, the West of Huangpu Street, Guangzhou, China. ${ }^{2}$ Department of Respiratory Medicine, Guangzhou First People's Hospital, Guangzhou Medical University, Panfu Road, Guangzhou, China. ${ }^{3}$ Department of Hepatobiliary Surgery, the Third Affiliated Hospital of Sun Yat-sen University, Tian He Road, Guangzhou, China. ${ }^{4}$ Department of Respiratory Medicine, Guangzhou Red Cross Hospital, Tong Fu Zhong Road, Guangzhou, China. ${ }^{5}$ Department of Clinic Laboratory, Guangzhou First People's Hospital, Guangzhou Medical University, Panfu Road, Guangzhou, China. ${ }^{6}$ Department of Clinic Laboratory, the First Affiliated Hospital of Sun Yat-sen University, Zhong Shan Er Road, Guangzhou, China.

Received: 26 August 2016 Accepted: 16 May 2017

Published online: 30 May 2017

\section{References}

1. Ying J, Lu J, Zong L, Li A, Pan R, Cheng C, et al. Molecular epidemiology and characterization of genotypes of Acinetobacter baumannii isolates from regions of South China. Jpn J Infect Dis. 2016;69:180-5.

2. El-Shazly S, Dashti A, Vali L, Bolaris M, Ibrahim AS. Molecular epidemiology and characterization of multiple drug-resistant (MDR) clinical isolates of Acinetobacter baumannii. Int J Infect Dis. 2015;41:42-9.

3. Hu FP, Guo Y, Zhu DM, Wang F, Jiang XF, Xu YC, et al. Resistance trends among clinical isolates in China reported from CHINET surveillance of bacterial resistance, 2005-2014. Clin Microbiol Infect. 2016;22 Suppl 1:S9-14.

4. Doi Y, Husain S, Potoski BA, McCurry KR, Paterson DL. Extensively drugresistant Acinetobacter baumannii. Emerg Infect Dis. 2009;15:980-2.

5. Li YJ, Pan CZ, Zhao ZW, Zhao ZX, Chen HL, Lu WB. Effects of a combination of amlodipine and imipenem on 42 clinical isolates of Acinetobacter baumannii obtained from a teaching hospital in Guangzhou, China. BMC Infect Dis. 2013;13:548. 
6. Batirel A, Balkan II, Karabay O, Agalar C, Akalin S, Alici O, et al. Comparison of colistin-carbapenem, colistin-sulbactam, and colistin plus other antibacterial agents for the treatment of extremely drug-resistant Acinetobacter baumannii bloodstream infections. Eur J Clin Microbiol Infect Dis. 2014:33:1311-22

7. Apisarnthanarak A, Warren DK. Intervention to limit transmission of extremely drug-resistant Acinetobacter baumannii in patients who underwent surgery. Clin Infect Dis. 2013;57:1215-6.

8. Peleg AY, Seifert H, Paterson DL. Acinetobacter baumannii: emergence of a successful pathogen. Clin Microbiol Rev. 2008;21:538-82.

9. Karah N, Sundsfjord A, Towner K, Samuelsen $\varnothing$. Insights into the global molecular epidemiology of carbapenem non-susceptible clones of Acinetobacter baumannii. Drug Resist Updat. 2012;15:237-47.

10. Fu Y, Zhou J, Zhou H, Yang Q, Wei Z, Yu Y, et al. Wide dissemination of OXA23-producing carbapenem-resistant Acinetobacter baumannii clonal complex 22 in multiple cities of China. J Antimicrob Chemother. 2010;65:644-50.

11. Ruan Z, Chen $Y$, Jiang $Y$, Zhou H, Zhou Z, Fu Y, et al. Wide distribution of CC92 carbapenem-resistant and OXA-23-producing Acinetobacter baumannii in multiple provinces of China. Int J Antimicrob Agents. 2013:42:322-8.

12. Zhong Q, Xu W, Wu Y, Xu H. Clonal spread of carbapenem non-susceptible Acinetobacter baumannii in an intensive care unit in a teaching hospital in China. Ann Lab Med. 2012;32:413-9.

13. He C, Xie Y, Zhang L, Kang M, Tao C, Chen Z, et al. Increasing imipenem resistance and dissemination of the ISAba1-associated blaOXA-23 gene among Acinetobacter baumannii isolates in an intensive care unit. J Med Microbiol. 2011;60:337-41.

14. Hamouda A, Evans BA, Towner KJ, Amyes SG. Characterization of epidemiologically unrelated Acinetobacter baumannii isolates from four continents by use of multilocus sequence typing, pulsed-field gel electrophoresis, and sequence- based typing of bla $a_{\mathrm{XX} \text {-51-like }}$ genes. J Clin Microbiol. 2010;48:2476-83

15. Evans BA, Hamouda A, Towner KJ, Amyes SG. OXA-51-like beta-lactamases and their association with particular epidemic lineages of Acinetobacter baumannii. Clin Microbiol Infect. 2008;14:268-75.

16. Zander E, Nemec A, Seifert H, Higgins PG. Association between $\beta$-LactamaseEncoding blaOXA-51 variants and DiversiLab rep-PCR-Based typing of Acinetobacter baumannii isolates. J Clin Microbiol. 2012;50:1900-4.

17. Clinical and Laboratory Standard Institute. Performance standards for antimicrobial susceptibility testing. Nineteenth informational supplement. CLSI Document M100 S22. Wayne: Clinical and Laboratory Standard Institute; 2012

18. Bartual SG, Seifert H, Hippler C, Luzon MA, Wisplinghoff H, Rodríguez-Valera F. Development of a multilocus sequence typing scheme for characterization of clinical isolates of Acinetobacter baumannii. J Clin Microbiol. 2005:43:4382-90.

19. Acinetobacter baumannii MLST databases. 2016. http://pubmlst.org/ abaumannii/. Accessed 3 Mar 2014.

20. eBURSTV3 algorithm. http://eburst.mlst.net/. Accessed 3 Mar 2014.

21. Héritier C, Poirel L, Fournier P-E, Claverie J-M, Raoult D, Nordmann P. Characterization of the naturally occurring Oxacillinase of Acinetobacter baumannii. Antimicrob Agents Chemother. 2005:49:4174-9.

22. American Thoracic Society, Infectious Diseases Society of America. Guidelines for the management of adults with hospital-acquired, ventilatorassociated, and healthcare-associated pneumonia. Am J Respir Crit Care Med. 2005:171:388-416.

23. Horan TC, Andrus M, Dudeck MA. CDC/NHSN surveillance definition of health care-associated infection and criteria for specific types of infections in the acute care setting. Am J Infect Control. 2008;36:309-32.

24. Lowings M, Ehlers MM, Dreyer AW, Kock MM. High prevalence of oxacillinases in clinical multidrug-resistant Acinetobacter baumannii isolates from the Tshwane region, South Africa - an update. BMC Infect Dis. 2015;15:521

25. Zhou Y, Wu X, Zhang X, Hu Y, Yang X, Yang Z, et al. Genetic characterization of ST195 and ST365 carbapenem-resistant Acinetobacter baumannii harboring blaOXA-23 in Guangzhou, China. Microb Drug Resist. 2015:21:386-90

26. Runnegar N, Sidjabat H, Goh HM, Nimmo GR, Schembri MA, Paterson DL. Molecular epidemiology of multidrug-resistant Acinetobacter baumannii in a single institution over a 10-year period. J Clin Microbiol. 2010;48:4051-6.

27. Pournaras S, Gogou V, Giannouli M, Dimitroulia E, Dafopoulou K, Tsakris A, et al. Single-locus-sequence-based typing of blaOXA-51-like genes for rapid assignment of Acinetobacter baumannii clinical isolates to international clonal lineages. J Clin Microbiol. 2014;52:1653-7.

28. Wang $H$, Guo $P$, Sun $H$, Wang $H$, Yang $Q$, Chen $M$, et al. Molecular epidemiology of clinical isolates of carbapenem-resistant Acinetobacter spp. From Chinese hospitals. Antimicrob Agents Chemother. 2007:51:4022-8.

29. Tsai H-T, Wang J-T, Chen CJ, Chang S-C. Association between antibiotic usage and subsequent colonization or infection of extensive drug-resistant Acinetobacter baumannii: a matched case-control study in intensive care units. Diagn Microbiol Infect Dis. 2008;62:298-305.

30. Dent LL, Marshall DR, Pratap S, Hulette RB. Multidrug resistant Acinetobacter baumannii: a descriptive study in a city hospital. BMC Infect Dis. 2010;10:196.

31. Zheng YL, Wan YF, Zhou LY, Ye ML, Liu S, Xu CQ, et al. Risk factors and mortality of patients with nosocomial carbapenem-resistant Acinetobacter baumannii pneumonia. Am J Infect Control. 2013;41:e1-5.

32. Li Y, Guo Q, Wang P, Zhu D, Ye X, Wu S, et al. Clonal dissemination of extensively drug-resistant Acinetobacter baumannii producing an OXA-23 $\beta$ lactamase at a teaching hospital in Shanghai, China. J Microbiol Immunol Infect. 2015;48:101-8.

33. Özgür ES, Horasan ES, Karaca K, Ersöz G, Naycı Atış S, Kaya A. Ventilatorassociated pneumonia due to extensive drug-resistant Acinetobacter baumannii: risk factors, clinical features, and outcomes. Am J Infect Control. 2014:42:206-8.

34. Chan MC, Chiu SK, Hsueh PR, Wang NC, Wang CC, Fang CT. Risk factors for healthcare-associated extensively drug-resistant Acinetobacter baumannil infections: a case-control study. PLoS One. 2014;9, e85973.

35. Yang Y-S, Lee Y-T, Huang TW, Sun J-R, Kuo S-C, Yang C-H, et al. Acinetobacter baumannii nosocomial pneumonia: is the outcome more favorable in nonventilated than ventilated patients? BMC Infect Dis. 2013;13:142.

36. Özvatan T, Akalın H, Sınırtaş M, Ocakoğlu G, Yılmaz E, Heper Y, et al. Nosocomial Acinetobacter pneumonia: treatment and prognostic factors in 356 cases. Respirology. 2016;21:363-9.

37. Sunenshine RH, Wright M-O, Maragakis LL, Harris AD, Song X, Hebden J, et al. Multidrug-resistant Acinetobacter infection mortality rate and length of hospitalization. Emerg Infect Dis. 2007;13:97-103.

\section{Submit your next manuscript to BioMed Central and we will help you at every step:}

- We accept pre-submission inquiries

- Our selector tool helps you to find the most relevant journal

- We provide round the clock customer support

- Convenient online submission

- Thorough peer review

- Inclusion in PubMed and all major indexing services

- Maximum visibility for your research

Submit your manuscript at www.biomedcentral.com/submit 